\title{
ON THE AIRY REPRODUCING KERNEL, SAMPLING SERIES, AND QUADRATURE FORMULA
}

\author{
ELI LEVIN ${ }^{1}$ AND DORON S. LUBINSKY ${ }^{2}$
}

\begin{abstract}
We determine the class of entire functions for which the Airy kernel (of random matrix theory) is a reproducing kernel. We deduce an Airy sampling series and quadrature formula. Our results are analogues of well known ones for the Bessel kernel. The need for these arises in investigating universality limits for random matrices at the soft edge of the spectrum.
\end{abstract}

\section{INTRODUCTION ${ }^{1}$}

Universality limits play a central role in random matrix theory [4], [17]. A recent new approach to these [14], [15], [16], [19] involves the reproducing kernel for a suitable space of entire functions . For universality in the bulk, the reproducing kernel is the familiar sine kernel, $\frac{\sin \pi t}{\pi t}$. It reproduces PaleyWiener space, the class of entire functions of exponential type at most $\pi$, that are square integrable along the real axis. More precisely, if $g$ is an entire function of exponential type at most $\pi$, and $g \in L_{2}(\mathbb{R})$, then for all complex $z$

$$
g(z)=\int_{-\infty}^{\infty} \frac{\sin \pi(t-z)}{\pi(t-z)} g(t) d t .
$$

For universality at the hard edge of the spectrum, one obtains instead the Bessel kernel. It is the reproducing kernel for a class of entire functions of exponential type $\leq 1$, satisfying a weighted integrability condition on the real line [16]. Both the sine and Bessel kernels have also been intensively studied within the context of sampling theory, as well as Lagrange interpolation series, and quadrature formula in approximation theory [6], [9], [10], [11], [12].

In investigating universality limits for random matrices at the soft edge of the spectrum, the authors needed to determine the class of entire functions for which the Airy kernel is a reproducing kernel. An extensive literature search did not turn up the desired results. While Paley-Wiener theorems have been obtained for some related Airy operators [21], and many identities for the Airy kernel are familiar in universality theory [2], [3], [5], [20], they do not readily yield the desired reproducing kernel formula. The associated sampling series, and quadrature formula, also could not be traced. It is

Date: January 20, 2009.

${ }^{1}$ Research supported by NSF grant DMS0400446 and US-Israel BSF grant 2004353 
the goal of this paper to present these. These will be an essential input to establishing universality at the soft edge of the spectrum for fairly general measures, in a future work.

Recall that the Airy kernel $\mathbb{A} i(\cdot, \cdot)$ of random matrix theory, is defined by

$$
\mathbb{A} i(a, b)= \begin{cases}\frac{A i(a) A i^{\prime}(b)-A i^{\prime}(a) A i(b)}{a-b}, & a \neq b, \\ A i^{\prime}(a)^{2}-a A i(a)^{2}, & a=b,\end{cases}
$$

where $A i$ is the Airy function, given on the real line by $[1,10.4 .32$, p. 447], $[18$, p. 53]

$$
A i(x)=\frac{1}{\pi} \int_{0}^{\infty} \cos \left(\frac{1}{3} t^{3}+x t\right) d t .
$$

The Airy function $A i$ is an entire function of order $\frac{3}{2}$, with only real negative zeros $\left\{a_{j}\right\}$, where

$$
0>a_{1}>a_{2}>a_{3}>\ldots
$$

and $[1,10.4 .94$, p. 450], [22, pp. 15-16]

$$
a_{j}=-[3 \pi(4 j-1) / 8]^{2 / 3}\left(1+O\left(\frac{1}{j^{2}}\right)\right)=-\left(\frac{3 \pi j}{2}\right)^{2 / 3}(1+o(1)) .
$$

Ai satisfies the differential equation

$$
A i^{\prime \prime}(z)-z A i(z)=0 .
$$

We note also that standard estimates for $A i$ show that $\mathbb{A} i(z, \cdot) \in L_{2}(\mathbb{R})$ for each fixed $z$..

Our main result is as follows:

\section{Theorem 1}

Let $g \in L_{2}(\mathbb{R})$ be the restriction to the real axis of an entire function. The following are equivalent:

(I) For all $z \in \mathbb{C}$,

$$
g(z)=\int_{-\infty}^{\infty} \mathbb{A} i(z, s) g(s) d s .
$$

(II) All the following are true:

(a) $g$ is an entire function of order at most $\frac{3}{2}$.

(b) There exists $L>0$ with the following property: whenever $0<\delta<\pi$, there exists $C_{\delta}$ such that for $|\arg (z)| \leq \pi-\delta$,

$$
|g(z)| \leq C_{\delta}(1+|z|)^{L}\left|e^{-\frac{2}{3} z^{3 / 2}}\right|
$$

(c)

$$
\sum_{j=1}^{\infty} \frac{\left|g\left(a_{j}\right)\right|^{2}}{\left|a_{j}\right|^{1 / 2}}<\infty
$$




\section{Remarks}

(a) For $|\arg (z)| \leq \pi-\delta$, with $|z| \rightarrow \infty$, [1, 10.4.59, p. 448], [18, p. 116]

$$
A i(z)=\frac{e^{-\frac{2}{3} z^{3 / 2}}}{2 \pi^{1 / 2} z^{1 / 4}}(1+o(1))
$$

so we may also formulate the bound (1.7) as

$$
|g(z)| \leq C_{\delta}(1+|z|)^{L+\frac{1}{4}}|A i(z)| \text {, for }|\arg (z)| \leq \pi-\delta .
$$

In fact, the representation (1.6) implies that (1.7) holds with $L=\frac{1}{2}$, as shown in the proof of Lemma 2.4.

(b) At first sight, the series condition (1.8) seems to follow from the other hypotheses, especially that $g \in L_{2}(\mathbb{R})$. If $g$ was of exponential type, this would be the case, but it is not obvious for entire functions of order larger than 1. At least, the series condition (1.8) is implied by a condition on the derivative of $g$ :

\section{Theorem 2}

If

$$
\int_{-\infty}^{0} \frac{\left|g^{\prime}(x)\right|^{2}}{1+|x|} d x<\infty
$$

together with the other requirements (II) (a), (b) of Theorem 1, then the series in (1.8) converges.

We can deduce an orthogonal sampling series, of the type associated with Kramer's lemma in signal processing and approximation theory [10], [12].

Note that $\left\{\frac{\mathbb{A} i\left(a_{j}, \cdot\right)}{\sqrt{\mathbb{A} i\left(a_{j}, a_{j}\right)}}\right\}_{j=1}^{\infty}$ is an orthonormal sequence in $L_{2}(\mathbb{R})$, as we shall show in Lemma 2.2 .

\section{Corollary 3}

Under the assumptions of Theorem 1(II), we have for all complex $z$,

$$
g(z)=\sum_{j=1}^{\infty} g\left(a_{j}\right) \frac{\mathbb{A} i\left(a_{j}, z\right)}{\mathbb{A} i\left(a_{j}, a_{j}\right)} .
$$

We may also deduce a quadrature formula:

\section{Corollary 4}

Assume that both $f$ and $g$ satisfy the assumptions of Theorem 1(II). Then

$$
\int_{-\infty}^{\infty}(f g)(x) d x=\sum_{j=1}^{\infty} \frac{(f g)\left(a_{j}\right)}{\mathbb{A} i\left(a_{j}, a_{j}\right)} .
$$


It is interesting that all the abscissa $\left\{a_{j}\right\}$ lie in $(-\infty, 0)$, while the integral extends over the whole real line. We note that in the Bessel kernel case, more comprehensively formulated quadrature formulae have been developed by Dryanov, Frappier, Grozev, Olivier, and Rahman [6], [8], [9], [10].

\section{Proofs}

Throughout, $C, C_{1}, C_{2}, \ldots$ denote constants indpendent of $n, z, x, t$. The same symbol does not necessarily denote the same constant in different occurrences. We begin with:

\section{Theorem 2.1}

For $a, b \in \mathbb{R}$,

$$
\int_{-\infty}^{\infty} \mathbb{A} i(a, s) \mathbb{A} i(s, b) d s=\mathbb{A} i(a, b)
$$

\section{Proof}

We shall use the results of Vu Kim Tuan [21] on the Airy integral transform. To avoid confusion with the Airy function, we denote this by $\mathcal{L}$. Thus, for $g \in L_{2}(\mathbb{R})$, we define, for each $R, S>0$,

$$
\mathcal{L}[g]_{R S}(x)=\int_{-S}^{R} A i(x+t) g(t) d t .
$$

As $R, S \rightarrow \infty$, this converges in $L_{2}(\mathbb{R})$ to a function $\mathcal{L}[g]$,

$$
\mathcal{L}[g](x)=\int_{-\infty}^{\infty} A i(x+t) g(t) d t
$$

Since $A i$ itself is not in $L_{2}(\mathbb{R})$, the integral does not converge necessarily for all $x$. It is is known [21, p. 525, Theorem 2] that this is an isometry on $L_{2}(\mathbb{R})$, so

$$
\int_{-\infty}^{\infty} \mathcal{L}[g](x)^{2} d x=\int_{-\infty}^{\infty} g(x)^{2} d x
$$

From this, and the relation $4 g h=(g+h)^{2}-(g-h)^{2}$, we immediately deduce a Parseval identity for $g, h \in L_{2}(\mathbb{R})$,

$$
\int_{-\infty}^{\infty} \mathcal{L}[g](x) \mathcal{L}[h](x) d x=\int_{-\infty}^{\infty} g(x) h(x) d x .
$$

$\mathcal{L}$ is also self-inversive. Thus if $g \in L_{1}(\mathbb{R})$, and (for example) $g$ is differentiable at $x$, then $[21$, p. 524 , Theorem 1$]$

$$
g(x)=\int_{-\infty}^{\infty} A i(x+t) \mathcal{L}[g](t) d t,
$$

where the integral is taken in a Cauchy principal value sense (integrate over $[-R, R]$ and let $R \rightarrow \infty)$. We also use a well known identity for the Airy 
kernel $[20$, p. 165, (4.5)]

$$
\mathbb{A} i(x, y)=\int_{0}^{\infty} A i(x+t) A i(y+t) d t
$$

for the Airy kernel. As shown there, this identity is an easy consequence of the differential equation (1.5). Let us now define, for a given $y$ and all $t$,

$$
f_{y}(t)=A i(y+t) \chi_{[0, \infty)}(t),
$$

where $\chi_{[0, \infty)}$ is the characteristic function of $[0, \infty)$. We can reformulate $(2.4)$ as

$$
\mathbb{A} i(x, y)=\mathcal{L}\left[f_{y}\right](x) .
$$

Here $f_{y}$ is certainly in $L_{1}(\mathbb{R}) \cap L_{2}(\mathbb{R})$, as $[1,(10.4 .59)$, p. 448]

$$
|A i(x)| \leq C(1+|x|)^{-1 / 2} \exp \left(-\frac{2}{3} x^{3 / 2}\right), x \in(0, \infty) .
$$

We now apply the Parseval identity (2.2):

$$
\int_{-\infty}^{\infty} \mathcal{L}\left[f_{y}\right](t) \mathcal{L}\left[f_{x}\right](t) d t=\int_{-\infty}^{\infty} f_{y}(t) f_{x}(t) d t
$$

Because of (2.5), this gives

$$
\int_{-\infty}^{\infty} \mathbb{A} i(t, y) \mathbb{A} i(t, x) d t=\int_{0}^{\infty} A i(y+t) A i(x+t) d t
$$

and (2.4) gives the result.

Our original proof of the above result started with the reproducing kernel identity for the Hermite weight, and then involved taking appropriately scaled limits. One uses the fact that Airy universality at the edge is known for the Hermite weight. This proof is longer and more computational, but uses more well known results.

We remind the reader that $[1,10.4 .94$, p. 450], [22, pp. 15-16]

$$
a_{j}=-[3 \pi(4 j-1) / 8]^{2 / 3}\left(1+O\left(\frac{1}{j^{2}}\right)\right)=-\left(\frac{3 \pi j}{2}\right)^{2 / 3}(1+o(1))
$$

So

$$
\left|a_{j}\right|^{3 / 2}-\left|a_{j-1}\right|^{3 / 2}=\frac{3 \pi}{2}+O\left(\frac{1}{j}\right) ;\left|a_{j}\right|-\left|a_{j-1}\right|=\pi\left|a_{j}\right|^{-1 / 2}(1+o(1)) .
$$

Moreover, [1, (10.4.96), p. 450], [22, p. 16]

$A i^{\prime}\left(a_{j}\right)=(-1)^{j-1} \pi^{-1 / 2}[3 \pi(4 j-1) / 8]^{1 / 6}(1+o(1))=(-1)^{j-1} \pi^{-1 / 2}\left|a_{j}\right|^{1 / 4}(1+o(1))$ and then (1.1) gives,

$$
\mathbb{A} i\left(a_{j}, a_{j}\right)=A i^{\prime}\left(a_{j}\right)^{2}=\frac{1}{\pi}\left|a_{j}\right|^{1 / 2}(1+o(1)) .
$$


In the sequel, we let

$$
e_{j}(z)=\frac{\mathbb{A} i\left(a_{j}, z\right)}{\sqrt{\mathbb{A} i\left(a_{j}, a_{j}\right)}}, j \geq 1,
$$

and also let $\mathcal{H}$ denote the Hilbert space which is the closure of the linear span of $\left\{e_{j}: j \geq 1\right\}$, with the usual inner product of $L_{2}(\mathbb{R})$, where complex valued functions are permitted:

$$
(f, g)=\int_{-\infty}^{\infty} f \bar{g}
$$

\section{Lemma 2.2}

(a) Let $\left\{a_{k}\right\}_{k=1}^{\infty}$ denote the zeros of $A i(z)$. Then

$$
\int_{-\infty}^{\infty} \mathbb{A} i\left(a_{j}, s\right) \mathbb{A} i\left(s, a_{k}\right) d s=\delta_{j, k} \mathbb{A} i\left(a_{j}, a_{j}\right) .
$$

(b) For any complex sequence $\left\{c_{k}\right\}_{k=1}^{\infty} \in \ell_{2}$,

$$
\int_{-\infty}^{\infty}\left|\sum_{j=1}^{\infty} c_{j} e_{j}\right|^{2}=\sum_{j=1}^{\infty}\left|c_{j}\right|^{2}
$$

\section{Proof}

(a) This follows from Theorem 2.1 and the fact (cf. (1.1)) that $\mathbb{A} i\left(a_{j}, a_{k}\right)=0$ when $j \neq k$.

(b) From (a), the sequence $\left\{e_{j}\right\}$ is an orthonormal sequence in $L_{2}(\mathbb{R})$. Then (2.11) is just the Parseval identity.

\section{Lemma 2.3}

Let $\beta \in(0,1)$. For $z \in \mathbb{C}$, let $a_{c}$ denote the closest zero of $A i$ to $\operatorname{Re} z$.

(a) For $z \in \mathbb{C}$ with $\operatorname{Re} z \geq 0$ or $|\operatorname{Im} z| \geq \beta|\operatorname{Re} z|$,

$$
\sum_{j=1}^{\infty} \frac{\left|\mathbb{A} i\left(a_{j}, z\right)\right|^{2}}{\mathbb{A} i\left(a_{j}, a_{j}\right)} \leq \frac{C}{1+|z|^{1 / 2}}|A i(z)|^{2} .
$$

If $\operatorname{Re} z<0$, we instead obtain

$$
\sum_{j=1}^{\infty} \frac{\left|\mathbb{A} i\left(a_{j}, z\right)\right|^{2}}{\mathbb{A} i\left(a_{j}, a_{j}\right)} \leq C|A i(z)|^{2}\left(|z|+\frac{1}{\left|z-a_{c}\right|^{2}}\right) .
$$

Moreover, the series converges uniformly for $z$ in compact subsets of $\mathbb{C}$.

(b) Let $g \in \mathcal{H}$. Then for all complex $z$,

$$
\begin{aligned}
& |g(z)| \leq C\|g\|_{L_{2}(\mathbb{R})} \times \\
& \times|A i(z)|\left\{\begin{array}{cc}
\frac{1}{(1+|z|)^{1 / 4}} & , \operatorname{Re} z \geq 0 \text { or }|\operatorname{Im} z| \geq \beta|\operatorname{Re} z| \\
|z|^{1 / 2}+\frac{1}{\left|z-a_{c}\right|} & , \operatorname{Re} z<0
\end{array} .\right.
\end{aligned}
$$




\section{Proof}

(a) Now from (1.1), $\mathbb{A} i\left(a_{j}, z\right)=A i(z) A i^{\prime}\left(a_{j}\right) /\left(z-a_{j}\right)$, so (2.9) gives

$$
\sum_{j=1}^{\infty} \frac{\left|\mathbb{A} i\left(a_{j}, z\right)\right|^{2}}{\mathbb{A} i\left(a_{j}, a_{j}\right)}=|A i(z)|^{2} \sum_{j=1}^{\infty} \frac{1}{\left|z-a_{j}\right|^{2}} .
$$

Here

$$
\begin{aligned}
& \sum_{j=1}^{\infty} \frac{1}{\left|z-a_{j}\right|^{2}} \\
\leq & \sum_{j:\left|a_{j}\right| \leq \frac{|z|}{2}} \frac{4}{|z|^{2}}+\sum_{j: \frac{|z|}{2} \leq\left|a_{j}\right| \leq 2|z|} \frac{1}{\left|z-a_{j}\right|^{2}}+\sum_{j:\left|a_{j}\right| \geq 2|z|} \frac{4}{a_{j}^{2}} \\
\leq & C|z|^{-1 / 2}+\sum_{j: \frac{|z|}{2} \leq\left|a_{j}\right| \leq 2|z|} \frac{1}{\left|z-a_{j}\right|^{2}},
\end{aligned}
$$

by (2.6) and some straightforward estimation. Here, if $\operatorname{Re} z \geq 0$, or $|\operatorname{Im} z| \geq$ $\beta|\operatorname{Re} z|$, we see that

$$
\begin{aligned}
& \sum_{j: \frac{|z|}{2} \leq\left|a_{j}\right| \leq 2|z|} \frac{1}{\left|z-a_{j}\right|^{2}} \\
\leq & C|z|^{1 / 2} \sum_{j: \frac{|z|}{2} \leq\left|a_{j}\right| \leq 2|z|} \frac{\left|a_{j}-a_{j-1}\right|}{\left|z-a_{j}\right|^{2}} \\
\leq & C|z|^{1 / 2} \int_{-\infty}^{0} \frac{d t}{|z-t|^{2}} \leq C|z|^{-1 / 2} .
\end{aligned}
$$

Instead, if $x=\operatorname{Re} z<0$, and $|\operatorname{Im} z|<\beta|\operatorname{Re} z|$, choose $k$ such that $x \in$ $\left(a_{k}, a_{k-1}\right)$. Then, as above,

$$
\begin{gathered}
\sum_{j: \frac{|z|}{2} \leq\left|a_{j}\right| \leq 2|z| \text { and }|j-k| \geq 2} \frac{1}{\left|z-a_{j}\right|^{2}} \\
\leq C|x|^{1 / 2} \int_{(-\infty, 0] \backslash\left(a_{k+1}, a_{k-2}\right)} \frac{d t}{|x-t|^{2}} \\
\leq C \frac{|x|^{1 / 2}}{\left|x-a_{k \pm 2}\right|} \leq C|x| \leq C|z| .
\end{gathered}
$$

This directly leads to (2.13). The uniform convergence follows by easy modification of the above estimates.

(b) Each $g \in \mathcal{H}$ has an orthonormal expansion

$$
g=\sum_{j=1}^{\infty} c_{j} e_{j}
$$


with

$$
\|g\|_{L_{2}(\mathbb{R})}=\left(\sum_{j=1}^{\infty}\left|c_{j}\right|^{2}\right)^{1 / 2} .
$$

Then the assertion follows directly from (a) and Cauchy-Schwarz.

\section{Lemma 2.4}

Let g satisfy the hypotheses (II) (a), (b), (c) of Theorem 1. Then

$$
g=\sum_{j=1}^{\infty} \frac{g\left(a_{j}\right)}{\sqrt{\mathbb{A} i\left(a_{j}, a_{j}\right)}} e_{j} .
$$

The series converges uniformly on compact sets, and $g \in \mathcal{H}$.

Proof

First note that

$$
H=\sum_{j=1}^{\infty} \frac{g\left(a_{j}\right)}{\sqrt{\mathbb{A} i\left(a_{j}, a_{j}\right)}} e_{j}
$$

satisfies

$$
\int_{-\infty}^{\infty}|H(s)|^{2} d s=\sum_{j=1}^{\infty} \frac{\left|g\left(a_{j}\right)\right|^{2}}{\mathbb{A} i\left(a_{j}, a_{j}\right)} \leq C \sum_{j=1}^{\infty} \frac{\left|g\left(a_{j}\right)\right|^{2}}{\left|a_{j}\right|^{1 / 2}}<\infty,
$$

in view of (2.11) and (2.9), and by our hypothesis (1.8). Moreover, $H \in \mathcal{H}$, and the series $H(z)$ converges to an entire function by the last lemma. If $\operatorname{Re} z \geq 0$ or $|\operatorname{Im} z| \geq|\operatorname{Re} z|$,

$$
|H(z)| \leq C \frac{|A i(z)|}{(1+|z|)^{1 / 4}},
$$

while otherwise,

$$
|H(z)| \leq C|A i(z)|\left(|z|^{1 / 2}+\frac{1}{\left|z-a_{c}\right|}\right),
$$

where $a_{c}$ is the closest zero of $A i$ to $\operatorname{Re} z$. Since $A i$ is entire of order $3 / 2$, these inequalities show that $H$ is also of order at most $3 / 2$. We assumed that the same is true of $g$. Next, let

$$
F(z)=(g(z)-H(z)) / A i(z) .
$$

As $H\left(a_{j}\right)=g\left(a_{j}\right)$ for each $j, F$ is entire. Being a ratio of entire functions of order $\leq 3 / 2, F$ is also of order $\leq 3 / 2[13$, p. 13, Theorem 1]. Also for $|\arg (z)| \geq \delta>0$, we have by (1.9), (2.18), and (2.19),

$$
|F(z)| \leq C(1+|z|)^{L+1 / 2} \text {. }
$$

By an easy application of the Phragmen-Lindelöf principle, it follows that $F$ is a polynomial. Indeed, we can apply the Phragmen-Lindelöf principle on sectors of opening a little larger than $\frac{2 \pi}{3}$, [13, Theorem 1, p. 37], to 
$F(z) /(z-a)^{L+1}$, where $a$ is outside the sector, and we may assume that $L$ is an integer. We deduce that throughout the plane,

$$
|F(z)| \leq C(1+|z|)^{L+1} .
$$

By Liouville's theorem, $F$ is a polynomial $P$, so that

$$
g(z)-H(z)=P(z) A i(z) .
$$

Let us assume that $P$ is of degree $k \geq 0$,

$$
P(z)=b z^{k}+\ldots, \text { where } k \geq 0, b \neq 0 .
$$

Here as $g \in L_{2}(\mathbb{R})$ and by $(2.17)$,

$$
\int_{-\infty}^{0}(g-H)^{2}<\infty
$$

We now use the asymptotic $[1,(10.4 .60)$, p. 448], [18, p. 103]

$$
A i(-x)=\pi^{-1 / 2} x^{-1 / 4} \cos \left(\frac{2}{3} x^{3 / 2}-\frac{1}{4} \pi\right)+o\left(x^{-1 / 4}\right), x \rightarrow \infty
$$

to deduce

$$
\begin{aligned}
\infty & >\int_{-\infty}^{0}(g-H)^{2}=\int_{-\infty}^{0} P^{2} A i^{2} \\
& =|b|^{2} \frac{1}{\pi}\left(\frac{3}{2}\right)^{4 k / 3-2 / 3} \int_{0}^{\infty}\left(\cos \left(t-\frac{\pi}{4}\right)+o(1)\right)^{2}(1+o(1))^{2} t^{4 k / 3-2 / 3} d t \\
& =\infty,
\end{aligned}
$$

so $b=0$, a contradiction. Thus $g=H$.

Proof that $(\mathrm{II}) \Rightarrow(\mathrm{I})$ in Theorem 1

For $m \geq 1$, let

$$
g_{m}(s)=\sum_{j=1}^{m} \frac{g\left(a_{j}\right)}{\sqrt{\mathbb{A} i\left(a_{j}, a_{j}\right)}} e_{j} .
$$

Let $x \in \mathbb{R}$. In view of Theorem 2.1,

$$
\int_{-\infty}^{\infty} \mathbb{A} i(s, x) g_{m}(s) d s=g_{m}(x) .
$$

Moreover, as $m \rightarrow \infty$, we have $g_{m}(x) \rightarrow g(x)$ pointwise by the previous lemma, as well as $g_{m} \rightarrow g$ in $L_{2}(\mathbb{R})$. Since $\int_{-\infty}^{\infty} \mathbb{A} i(s, x)^{2} d s<\infty$, (for example, by Theorem 2.1), we can just let $m \rightarrow \infty$ in both sides of (2.20). This gives (1.6) for real $z$, and analytic continuation gives it for all $z$.

\section{Proof that $(\mathrm{I}) \Rightarrow(\mathrm{II})$ in Theorem 1}

The reproducing kernel property (1.6) gives

$$
\frac{g\left(a_{j}\right)}{\sqrt{\mathbb{A} i\left(a_{j}, a_{j}\right)}}=\int_{-\infty}^{\infty} g(s) e_{j}(s) d s=\left(g, e_{j}\right) .
$$


Bessel's inequality for orthonormal expansions in $L_{2}(\mathbb{R})$ gives

$$
\sum_{j=1}^{\infty} \frac{\left|g\left(a_{j}\right)\right|^{2}}{\mathbb{A} i\left(a_{j}, a_{j}\right)} \leq \int_{-\infty}^{\infty} g^{2}<\infty .
$$

So (1.8) follows (recall (2.9)). Next, we may define the sampling series $H$ as in (2.16), and it has the properties (2.17) - (2.19). We use this to show $g=H$ and hence verify the growth condition (1.9). First, observe that for fixed $s$ not a zero of $A i$, the function

$$
G(z)=\mathbb{A} i(s, z)
$$

satisfies all the hypotheses (II) (a), (b), (c) of Theorem 1. Indeed, it belongs to $L_{2}(\mathbb{R})$ by Theorem 2.1 , while

$$
G\left(a_{j}\right)=\frac{A i^{\prime}\left(a_{j}\right) A i(s)}{s-a_{j}},
$$

so (2.9) shows that

$$
\sum_{j=1}^{\infty} \frac{\left|G\left(a_{j}\right)\right|^{2}}{\left|a_{j}\right|^{1 / 2}} \leq C|A i(s)|^{2} \sum_{j=1}^{\infty} \frac{1}{\left|a_{j}-s\right|^{2}}<\infty .
$$

Finally, the growth condition and order conditions are immediate. By Lemma 2.4, applied to $g=G$,

$$
\mathbb{A} i(s, x)=\sum_{j=1}^{\infty} \frac{\mathbb{A} i\left(a_{j}, x\right)}{\sqrt{\mathbb{A} i\left(a_{j}, a_{j}\right)}} e_{j}(s)=\sum_{j=1}^{\infty} e_{j}(x) e_{j}(s),
$$

and hence for real $x$,

$$
\int_{-\infty}^{\infty} \mathbb{A} i(s, x)^{2} d s=\sum_{j=1}^{\infty} \frac{\mathbb{A} i\left(a_{j}, x\right)^{2}}{\mathbb{A} i\left(a_{j}, a_{j}\right)}=\sum_{j=1}^{\infty} e_{j}^{2}(x) .
$$

Then for $n \geq 1$, and real $x$, our hypothesis (1.6) gives

$$
\begin{aligned}
& \left|g(x)-\sum_{j=1}^{n} \frac{g\left(a_{j}\right)}{\sqrt{\mathbb{A} i\left(a_{j}, a_{j}\right)}} e_{j}(x)\right| \\
= & \left|\int_{-\infty}^{\infty} g(s)\left\{\mathbb{A} i(s, x)-\sum_{j=1}^{n} e_{j}(s) e_{j}(x)\right\} d s\right| \\
\leq & \left\{\int_{-\infty}^{\infty} g(s)^{2} d s\right\}^{1 / 2}\left\{\int_{-\infty}^{\infty} \mathbb{A} i(s, x)^{2} d s-\sum_{j=1}^{n} e_{j}^{2}(x)\right\}^{1 / 2} \\
\rightarrow & 0, n \rightarrow \infty .
\end{aligned}
$$

(We used orthonormality in the second last line). Thus $g=H$ on the real line, and by analytic continuation throughout the plane. Since $H$ is of order $\leq \frac{3}{2}$ and satisfies (II)(b) of Theorem 1 (recall (2.18) and (2.19)), we are 
done.

\section{Proof of Theorem 2}

By the fundamental theorem of calculus, and some simple estimation,

$$
\left|g\left(a_{j}\right)\right|^{2} \leq \inf _{\left[a_{j+1}, a_{j}\right]}|g|^{2}+2 \int_{a_{j+1}}^{a_{j}}\left|g g^{\prime}\right| .
$$

Using (2.7), we can continue this as

$$
\begin{aligned}
\frac{\left|g\left(a_{j}\right)\right|^{2}}{\left|a_{j}\right|^{1 / 2}} & \leq C\left(a_{j}-a_{j+1}\right) \inf _{\left[a_{j+1}, a_{j}\right]}|g|^{2}+C \int_{a_{j+1}}^{a_{j}} \frac{\left|g g^{\prime}\right|(x)}{1+|x|^{1 / 2}} d x \\
& \leq C \int_{a_{j+1}}^{a_{j}}|g|^{2}+C \int_{a_{j+1}}^{a_{j}} \frac{\left|g g^{\prime}\right|(x)}{1+|x|^{1 / 2}} d x .
\end{aligned}
$$

Adding over $j$ gives

$$
\begin{aligned}
\sum_{j=1}^{\infty} \frac{\left|g\left(a_{j}\right)\right|^{2}}{\left|a_{j}\right|^{1 / 2}} & \leq C \int_{-\infty}^{0}|g|^{2}+C \int_{-\infty}^{0} \frac{\left|g g^{\prime}\right|(x)}{1+|x|^{1 / 2}} d x \\
& \leq C \int_{-\infty}^{0}|g|^{2}+C\left(\int_{-\infty}^{0}|g|^{2}\right)^{1 / 2}\left(\int_{-\infty}^{0} \frac{\left|g^{\prime}\right|^{2}(x)}{1+|x|} d x\right)^{1 / 2},
\end{aligned}
$$

by Cauchy-Schwarz. So (1.8) is satisfied.

\section{Proof of Corollary 3}

This follows from Lemma 2.4.

\section{Proof of Corollary 4}

This follows easily from Corollary 3 and the orthonormality of $\left\{e_{j}\right\}$.

\section{Acknowledgement}

The authors acknowledge very helpful comments from the referee that simplified some of the proofs.

\section{REFERENCES}

[1] Abramowitz and I. A. Stegun, Handbook of Mathematical Functions, Dover, New York, 1965.

[2] J. Baik, L. Li, T. Kriecherbauer, K. McLaughlin, C. Tomei, Proceedings of the Conference on Integrable Systems, Random Matrices and Applications, to appear in Contemporary Mathematics, American Mathematical Society.

[3] E.L. Basor and H. Widom, Determinants of Airy Operators and Applications to Random Matrices, J. Statistical. Phys., 96 (1999) 1-20.

[4] P. Deift, Orthogonal Polynomials and Random Matrices: A Riemann-Hilbert Approach, Courant Institute Lecture Notes, Vol. 3, New York University Pres, New York, 1999.

[5] P. Deift, T. Kriecherbauer, K. T-R McLaughlin, S. Venakides, and X. Zhou, Uniform Asymptotics for Polynomials Orthogonal with respect to Varying Exponential Weights 
and Applications to Universality Questions in Random Matrix Theory, Communications in Pure and Applied Mathematics, 52(1999), 1335-1425.

[6] D.P. Dryanov, M.A. Qazi, Q. I. Rahman, Entire Functions of Exponential Type in Approximation Theory, (in) Constructive Theory of Functions, Varna 2002, (ed. B. Bojaov), DARBA, Sofia, 2003, pp. 86-135.

[7] P.J. Forrester, The Spectrum Edge of Random Matrix Ensembles, Nucl. Phys. B, 402(1993), 709-728.

[8] C. Frappier and P. Olivier, A Quadrature Formula Involving Zeros of Bessel Functions, Math. Comp., 60(1993), 303-316.

[9] G.R. Grozev, Q.I. Rahman, A Quadrature Formula involving Zeros of Bessel Functions as nodes, Math. Comp., 64(1995), 715-725.

[10] G.R. Grozev, Q.I. Rahman, Lagrange Interpolation in the Zeros of Bessel functions by Entire Functions of Exponential Type and Mean Convergence, Methods and Applications of Analysis, 3(1996), 46-79.

[11] J.R. Higgins, An Interpolation Series Associated with the Bessel-Hankel Transform, J. London Math. Soc., 5(1972), 707-714.

[12] J.R. Higgins, Sampling Theory in Fourier and Signal Analysis: Foundations, Oxford University Press, Oxford, 1996.

[13] B. Ya. Levin, in collaboration with Yu. Lyubarskii, M. Sodin, V. Tkachenko, Lectures on Entire Functions, Translations of Mathematical Monographs, Vol. 150, American Mathematical Society, Providence, 1996.

[14] Eli Levin and D.S. Lubinsky, Universality Limits in the Bulk for Varying Measures, Advances in Mathematics, 219(2008), 743-779.

[15] D.S. Lubinsky, Universality Limits in the Bulk for Arbitrary Measures on Compact Sets, J. de'Analyse de Mathematique, 106(2008), 373-394.

[16] D.S. Lubinsky, Universality Limits at the Hard Edge of the Spectrum for Measures with Compact Support, to appear in International Mathematics Research Notices.

[17] M.L. Mehta, Random Matrices, 2nd edn., Academic Press, Boston, 1991.

[18] F.W.J. Olver, Asymptotics and Special Functions, Academic Press, San Diego, 1974.

[19] B. Simon, The Christoffel-Darboux Kernel, to appear.

[20] C.A. Tracy and H. Widom, Level-Spacing Distributions and the Airy Kernel, Comm. Math. Phys., 159(1994), 151-174.

[21] Vu Kim Tuan, Airy Integral Transform and the Paley-Wiener Theorem, (in) Transform Methods and Special Functions, Varna, Bulgarian Academy of Sciences, 1998, pp. 523-531.

[22] O. Vallée and M. Soares, Airy Functions and Applications to Physics, World Scientific, Singapore, 2004.

${ }^{1}$ Mathematics Department, The Open University of Israel, P.O. Box 808, RaAnana 43107, Israel., ${ }^{2}$ School of Mathematics, Georgia Institute of TechNology, Atlanta, GA 30332-0160, USA., ${ }^{1}$ Lubinsky@Math.Gatech.edu, ${ }^{2}$ Elile@openu.AC.IL

School of Mathematics, Georgia Institute of Technology, Atlanta, GA 30332-0160, USA., LUBINSKY@MATH.GATECH.EDU 